\title{
GRAPH CLASSES GENERATED BY MYCIELSKIANS
}

\author{
Mieczysław Borowiecki $^{a}$, Piotr Borowiecki ${ }^{b, 1}$ \\ Ewa Drgas-Burchardi ${ }^{a}$ And ElżBIEta Sidorowicz ${ }^{a}$ \\ ${ }^{a}$ Institute of Mathematics \\ University of Zielona Góra \\ Prof. Z. Szafrana 4a, 65-516 Zielona Góra, Poland \\ ${ }^{b}$ Faculty of Electronics, Telecommunications and Informatics \\ Gdańsk University of Technology \\ Narutowicza 11/12, 80-233 Gdańsk, Poland \\ e-mail: m.borowiecki@wmie.uz.zgora.pl \\ pborowie@eti.pg.gda.pl \\ e.drgas-burchardt@wmie.uz.zgora.pl \\ e.sidorowicz@wmie.uz.zgora.pl
}

\begin{abstract}
In this paper we use the classical notion of weak Mycielskian $M^{\prime}(G)$ of a graph $G$ and the following sequence: $M_{0}^{\prime}(G)=G, M_{1}^{\prime}(G)=M^{\prime}(G)$, and $M_{n}^{\prime}(G)=M^{\prime}\left(M_{n-1}^{\prime}(G)\right)$, to show that if $G$ is a complete graph of order $p$, then the above sequence is a generator of the class of $p$-colorable graphs. Similarly, using Mycielskian $M(G)$ we show that analogously defined sequence is a generator of the class consisting of graphs for which the chromatic number of the subgraph induced by all vertices that belong to at least one triangle is at most $p$. We also address the problem of characterizing the latter class in terms of forbidden graphs.
\end{abstract}

Keywords: Mycielski graphs, graph coloring, chromatic number.

2010 Mathematics Subject Classification: 05C15, 05C75, 68R10, 05C60.

\section{REFERENCES}

[1] A.J. Berger, I. Broere, S.J.T. Moagi and P. Mihók, Meet- and join-irreducibility of additive hereditary properties of graphs, Discrete Math. 251 (2002) 11-18. doi:10.1016/S0012-365X(01)00323-5

\footnotetext{
${ }^{1}$ Partially supported under Ministry of Science and Higher Education (Poland) subsidy for Gdańsk University od Technology.
} 
[2] M. Borowiecki, I. Broere, M. Frick, P. Mihók and G. Semanišin, A survey of hereditary properties of graphs, Discuss. Math. Graph Theory 17 (1997) 5-50. doi:10.7151/dmgt.1037

[3] P. Borowiecki, Computational aspects of greedy partitioning of graphs, J. Comb. Optim. 35 (2018) 641-665. doi:10.1007/s10878-017-0185-2

[4] B. Brešar, J. Ferme, S. Klavžar and D.F. Rall, A survey on packing colorings, Discuss. Math. Graph Theory 40 (2020) 923-970. doi:10.7151/dmgt.2320

[5] B. Brešar, S. Klavžar, D.F. Rall and K. Wash, Packing chromatic number versus chromatic and clique number, Aequationes Math. 92 (2018) 497-513. doi:10.1007/s00010-017-0520-9

[6] M. Caramia and P. Dell'Olmo, A lower bound on the chromatic number of Mycielski graphs, Discrete Math. 235 (2001) 79-86. doi:10.1016/S0012-365X(00)00261-2

[7] G.J. Chang, L. Huang and X. Zhu, Circular chromatic number of Mycielski's graphs, Discrete Math. 205 (1999) 23-37. doi:10.1016/S0012-365X(99)00033-3

[8] M. Cropper, A. Gyárfás and J. Lehel, Hall ratio of the Mycielski graphs, Discrete Math. 306 (2006) 1988-1990. doi:10.1016/j.disc.2005.09.020

[9] R. Diestel, Graph Theory (Springer, Berlin, 1997).

[10] T. Došlić, Mycielskians and matchings, Discuss. Math. Graph Theory 25 (2005) 261-266. doi:10.7151/dmgt.1279

[11] D.C. Fisher, Fractional colorings with large denominators, J. Graph Theory 20 (1995) 403-409. doi:10.1002/jgt.3190200403

[12] D.C. Fisher, P.A. McKenna and E.D. Boyer, Hamiltonicity, diameter, domination, packing, and biclique partitions of Mycielski's graphs, Discrete Appl. Math. 84 (1998) 93-105. doi:10.1016/S0166-218X(97)00126-1

[13] Y.S. Kwon, J. Lee and Z. Zhang, Edge-chromatic numbers of Mycielski graphs, Discrete Math. 312 (2012) 1222-1225.

doi:10.1016/j.disc.2011.12.011

[14] M. Larsen, J. Propp and D. Ullman, The fractional chromatic number of Mycielski's graphs, J. Graph Theory 19 (1995) 411-416. doi:10.1002/jgt.3190190313

[15] W. Li, J. Wu, P.C.B. Lam and G. Gu, Several parameters of generalized Mycielskians, Discrete Appl. Math. 154 (2006) 1173-1182. doi:10.1016/j.dam.2005.11.001 
[16] D.D.-F. Liu, Circular chromatic number for iterated Mycielski graphs, Discrete Math. 285 (2004) 335-340.

doi:10.1016/j.disc.2004.01.020

[17] J. Mycielski, Sur le coloriage des graphes, Colloq. Math. 3 (1955) 161-162. doi:10.4064/cm-3-2-161-162

[18] H.P. Patil and R. Pandiya Raj, On the total graph of Mycielski graphs, central graphs and their covering numbers, Discuss. Math. Graph Theory 33 (2013) 361-371. doi:10.7151/dmgt.1670

[19] G. Simons, C. Tardif and D. Wehlau, Generalised Mycielski graphs, signature systems, and bounds on chromatic number, J. Combin. Theory Ser. B 122 (2017) 776-793.

doi:10.1016/j.jctb.2016.09.007

[20] D.B. West, Introduction to Graph Theory (Prentice Hall, Upper Saddle River, 2001).

Received 31 May 2020

Revised 10 July 2020

Accepted 10 July 2020 